\title{
Interval-valued Pythagorean fuzzy Einstein hybrid weighted averaging aggregation operator and their application to group decision making
}

\author{
Khaista Rahman ${ }^{1} \cdot$ Saleem Abdullah ${ }^{2} \cdot$ Asad Ali $^{1} \cdot$ Fazli Amin ${ }^{1}$
}

Received: 15 May 2017 / Accepted: 26 May 2018 / Published online: 5 June 2018

(c) The Author(s) 2018

\begin{abstract}
The objective of the present work is divided into two folds. Firstly, interval-valued Pythagorean fuzzy Einstein hybrid weighted averaging aggregation operator has been introduced along with their several properties, namely idempotency, boundedness and monotonicity. Secondly, we apply the proposed operator to deal with multi-attribute group decision-making problem under Pythagorean fuzzy information. For this, we construct an algorithm for multi-attribute group decision making. At the last, we construct a numerical example for multi-attribute group decision making. The main advantage of using the proposed operator is that this operator provides more accurate and precise results is compared to the existing methods.
\end{abstract}

Keywords IVPFS $\cdot$ IVPFEHWA averaging operator $\cdot$ MAGDM problems

\section{Introduction}

Multi-criteria group decision making is one of the successful processes for finding the optimal alternative from all the feasible alternatives according to some criteria or attributes. Traditionally, it has been generally assumed that all the information that access the alternative in terms of criteria and their corresponding weights are expressed in the form of crisp numbers. But most of the decisions in the real-life situations are taken in the environment where the goals and constraints are generally imprecise or vague in nature. In order to handle the uncertainties and fuzziness intuitionistic fuzzy set [1] theory is one of the successful extensions of the fuzzy set theory [2], which is characterized by the degree of membership and degree of non-membership has been presented. After the

Khaista Rahman

khaista355@yahoo.com

Saleem Abdullah

saleemabdullah81@yahoo.com

Asad Ali

asad_maths@hu.edu.pk

Fazli Amin

fazliamin@hu.edu.pk

1 Department of Mathematics, Hazara University, Mansehra, KPK, Pakistan

2 Department of Mathematics, Abdul Wali Khan University Mardan, Mardan, KPK, Pakistan successful and positive applications of intuitionistic fuzzy set, aggregation operators become more interesting topic for research. Thus, many scholars in [3-16] developed several aggregation operators for group decision making using intuitionistic fuzzy information.

However, there are many cases where the decision maker may provide the degree of membership and nonmembership of a particular attribute in such a way that their sum is greater than one. To solve these types of problems, Yager [17,18] introduced the concept of another set called Pythagorean fuzzy set. Pythagorean fuzzy set is more powerful tool to solve uncertain problems. Like intuitionistic fuzzy aggregation operators, Pythagorean fuzzy aggregation operators are also become an interesting and important area for research, after the advent of Pythagorean fuzzy set theory. Several researchers in [19-28] introduced many aggregation operators for decision using Pythagorean fuzzy information.

But, in some real decision-making problems, due to insufficiency in available information, it may be difficult for decision makers to exactly quantify their opinions with a crisp number, but they can be represented by an interval number within $[0,1]$. Therefore, it is so important to present the idea of interval-valued Pythagorean fuzzy sets, which permit the membership degrees and non- membership degrees to a given set to have an interval value. Thus in [29] Peng and Yang introduced the concept of interval-valued Pythagorean fuzzy set. Rahman et al. [30-33] introduced many aggregation operators using interval-valued Pythagorean fuzzy

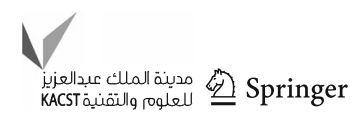


numbers and applied them to multi-attribute group decision making.

Thus, keeping the advantages of these operators, in this paper, we introduce the notion of interval-valued Pythagorean fuzzy Einstein hybrid weighted averaging operator. Moreover, we introduce some of their basic properties such as idempotency, boundedness and monotonicity. This motivation comes from [32], in which the authors introduced the notion of IVPFEWA operator and IVPFEOWA operator and applied them to group decision making. But in this paper we introduce the notion of IVPFEHWA operator, which is the generalization of the above mention operators.

The remainder of this paper is structured as follows. In Sect. "Preliminaries", we give some basic definitions and results which will be used in our later sections. In Sect. "Interval-valued Pythagorean fuzzy Einstein hybrid weighted averaging aggregation operator", we introduce the notion of interval-valued Pythagorean fuzzy Einstein hybrid weighted averaging operator. In Sect. "An approach to multiple attribute group decision-making problems based on intervalvalued Pythagorean fuzzy information", we apply the proposed operator to multi-attribute group decisionmaking problem with Pythagorean fuzzy information. In Sect. "Illustrative example", we develop a numerical example. In Sect. "Conclusion”, we have conclusion.

\section{Preliminaries}

Definition $1 \quad[17,18]$ Let $K$ be a fixed set, then a Pythagorean fuzzy set can be defined as:

$P=\left\{\left\langle k, u_{P}(k), v_{P}(k)\right\rangle \mid k \in K\right\}$,

where $u_{P}(k): P \rightarrow[0,1], v_{P}(k): K \rightarrow[0,1]$ are called membership function and non-membership function, respectively, with condition $0 \leq\left(u_{P}(k)\right)^{2}+\left(v_{P}(k)\right)^{2} \leq 1$, for all $k \in K$.

Let

$\pi_{P}(k)=\sqrt{1-u_{P}^{2}(k)-v_{P}^{2}(k)}$.

Then, it is called the Pythagorean fuzzy index of $k \in K$, with condition $0 \leq \pi_{P}(k) \leq 1$, for every $k \in K$.

Definition 2 [29] Let $K$ be a fixed set, then an interval-valued Pythagorean fuzzy set can be defined as:

$I=\left\{\left\langle k, u_{I}(k), v_{I}(k)\right\rangle \mid k \in K\right\}$,

where

$u_{I}(k)=\left[u_{I}^{a}(k), u_{I}^{b}(k)\right] \subset[0,1]$, and

$v_{I}(k)=\left[v_{I}^{a}(k), v_{I}^{b}(k)\right] \subset[0,1]$.

Also

$u_{I}^{a}(k)=\inf \left(u_{I}(k)\right)$,

$u_{I}^{b}(k)=\sup \left(u_{I}(k)\right)$,

$v_{I}^{a}(k)=\inf \left(v_{I}(k)\right)$,

$v_{I}^{b}(k)=\sup \left(v_{I}(k)\right)$,

and

$0 \leq\left(u_{I}^{b}(k)\right)^{2}+\left(v_{I}^{b}(k)\right)^{2} \leq 1$.

If

$\pi_{I}(k)=\left[\pi_{I}^{a}(k), \pi_{I}^{b}(k)\right]$, for all $k \in K$.

Then, it is called the interval-valued Pythagorean fuzzy index of $k$ to $I$, where

$\pi_{I}^{a}(k)=\sqrt{1-\left(u_{I}^{b}(k)\right)^{2}-\left(v_{I}^{b}(k)\right)^{2}}$,

and

$\pi_{I}^{b}(k)=\sqrt{1-\left(u_{I}^{a}(k)\right)^{2}-\left(v_{I}^{a}(k)\right)^{2}}$.

Definition 3 [29] Let $\lambda=\left(\left[u_{\lambda}, v_{\lambda}\right],\left[x_{\lambda}, y_{\lambda}\right]\right)$ be an IVPFN, then the score function and accuracy function of $\lambda$ can be defined as follows, respectively:

$s(\lambda)=\frac{1}{2}\left[\left(u_{\lambda}\right)^{2}+\left(v_{\lambda}\right)^{2}-\left(x_{\lambda}\right)^{2}-\left(y_{\lambda}\right)^{2}\right]$,

and

$h(\lambda)=\frac{1}{2}\left[\left(u_{\lambda}\right)^{2}+\left(v_{\lambda}\right)^{2}+\left(x_{\lambda}\right)^{2}+\left(y_{\lambda}\right)^{2}\right]$.

If $\lambda_{1}$ and $\lambda_{2}$ are two IVPFNs, then

1. If $s\left(\lambda_{1}\right) \prec s\left(\lambda_{2}\right)$, then $\lambda_{1} \prec \lambda_{2}$.

2 . If $s\left(\lambda_{1}\right)=s\left(\lambda_{2}\right)$, then we have the following three conditions.

1) If $h\left(\lambda_{1}\right)=h\left(\lambda_{2}\right)$, then $\lambda_{1}=\lambda_{2}$.

2) If $h\left(\lambda_{1}\right) \prec h\left(\lambda_{2}\right)$, then $\lambda_{1} \prec \lambda_{2}$.

3) If $h\left(\lambda_{1}\right) \succ h\left(\lambda_{2}\right)$, then $\lambda_{1} \succ \lambda_{2}$.

Definition 4 [32] Let $\lambda=([u, v],[x, y]), \lambda_{1}=\left(\left[u_{1}, v_{1}\right]\right.$, $\left.\left[x_{1}, y_{1}\right]\right), \lambda_{2}=\left(\left[u_{2}, v_{2}\right],\left[x_{2}, y_{2}\right]\right)$ are three IVPFNs, and $\delta \succ 0$, then some Einstein operations for $\lambda, \lambda_{1}, \lambda_{2}$ can be defined as follows: 
1.

$$
\begin{aligned}
\lambda_{1} \oplus_{\varepsilon} \lambda_{2}= & \left(\left[\frac{\sqrt{u_{1}^{2}+u_{2}^{2}}}{\sqrt{1+u_{1}^{2} u_{2}^{2}}}, \frac{\sqrt{v_{1}^{2}+v_{2}^{2}}}{\sqrt{1+v_{1}^{2} v_{2}^{2}}}\right],\right. \\
& {\left[\frac{x_{1} x_{2}}{\sqrt{1+\left(1-x_{1}^{2}\right)\left(1-x_{2}^{2}\right)}},\right.} \\
& \left.\left.\frac{y_{1} y_{2}}{\sqrt{1+\left(1-y_{1}^{2}\right)\left(1-y_{2}^{2}\right)}}\right]\right)
\end{aligned}
$$

2.

$$
\begin{aligned}
\lambda_{1} \otimes_{\varepsilon} \lambda_{2}= & \left(\left[\frac{u_{1} u_{2}}{\sqrt{1+\left(1-u_{1}^{2}\right)\left(1-u_{2}^{2}\right)}},\right.\right. \\
& \left.\frac{v_{1} v_{2}}{\sqrt{1+\left(1-v_{1}^{2}\right)\left(1-v_{2}^{2}\right)}}\right], \\
& {\left.\left[\frac{\sqrt{x_{1}^{2}+x_{2}^{2}}}{\sqrt{1+x_{1}^{2} x_{2}^{2}}}, \frac{\sqrt{y_{1}^{2}+y_{2}^{2}}}{\sqrt{1+y_{1}^{2} y_{2}^{2}}}\right]\right) }
\end{aligned}
$$

3.

$$
\begin{aligned}
\delta \lambda= & \left(\left[\frac{\sqrt{\left(1+u^{2}\right)^{\delta}-\left(1-u^{2}\right)^{\delta}}}{\sqrt{\left(1+u^{2}\right)^{\delta}+\left(1-u^{2}\right)^{\delta}}},\right.\right. \\
& \left.\frac{\sqrt{\left(1+v^{2}\right)^{\delta}-\left(1-v^{2}\right)^{\delta}}}{\sqrt{\left(1+v^{2}\right)^{\delta}+\left(1-v^{2}\right)^{\delta}}}\right], \\
& {\left.\left[\frac{\sqrt{2\left(x^{2}\right)^{\delta}}}{\sqrt{\left(2-x^{2}\right)^{\delta}+\left(x^{2}\right)^{\delta}}}, \frac{\sqrt{2\left(y^{2}\right)^{\delta}}}{\sqrt{\left(2-y^{2}\right)^{\delta}+\left(y^{2}\right)^{\delta}}}\right]\right) }
\end{aligned}
$$

4.

$$
\begin{aligned}
\lambda^{\delta}= & \left(\left[\frac{\sqrt{2\left(u^{2}\right)^{\delta}}}{\sqrt{\left(2-u^{2}\right)^{\delta}+\left(u^{2}\right)^{\delta}}}, \frac{\sqrt{2\left(v^{2}\right)^{\delta}}}{\sqrt{\left(2-v^{2}\right)^{\delta}+\left(v^{2}\right)^{\delta}}}\right],\right. \\
& {\left[\frac{\sqrt{\left(1+x^{2}\right)^{\delta}-\left(1-x^{2}\right)^{\delta}}}{\sqrt{\left(1+x^{2}\right)^{\delta}+\left(1-x^{2}\right)^{\delta}}},\right.} \\
& \left.\left.\frac{\sqrt{\left(1+y^{2}\right)^{\delta}-\left(1-y^{2}\right)^{\delta}}}{\sqrt{\left(1+y^{2}\right)^{\delta}+\left(1-y^{2}\right)^{\delta}}}\right]\right)
\end{aligned}
$$

Definition 5 [32] Let $\lambda_{j}=\left(\left[u_{j}, v_{j}\right],\left[x_{j}, y_{j}\right]\right)(j=1,2,3$, $\ldots, n)$ be the collection of IVPFVs, then IVPFEWA operator can be defined as:

$$
\begin{aligned}
& \operatorname{IVPFEWA}_{w}\left(\lambda_{1}, \lambda_{2}, \lambda_{3}, \ldots, \lambda_{n}\right) \\
& =\left(\begin{array}{l}
{\left[\frac{\sqrt{\prod_{j=1}^{n}\left(1+u_{\lambda_{j}}^{2}\right)^{w_{j}}-\prod_{j=1}^{n}\left(1-u_{\lambda_{j}}^{2}\right)^{w_{j}}}}{\sqrt{\prod_{j=1}^{n}\left(1+u_{\lambda_{j}}^{2}\right)^{w_{j}}+\prod_{j=1}^{n}\left(1-u_{\lambda_{j}}^{2}\right)^{w_{j}}}}, \frac{\sqrt{\prod_{j=1}^{n}\left(1+v_{\lambda_{j}}^{2}\right)^{w_{j}}-\prod_{j=1}^{n}\left(1-v_{\lambda_{j}}^{2}\right)^{w_{j}}}}{\sqrt{\prod_{j=1}^{n}\left(1+v_{\lambda_{j}}^{2}\right)^{w_{j}}+\prod_{j=1}^{n}\left(1-v_{\lambda_{j}}^{2}\right)^{w_{j}}}}\right],} \\
{\left[\frac{\sqrt{2 \prod_{j=1}^{n}\left(x_{\lambda_{j}}^{2}\right)^{w_{j}}}}{\sqrt{\prod_{j=1}^{n}\left(2-x_{\lambda_{j}}^{2}\right)^{w_{j}}+\prod_{j=1}^{n}\left(x_{\lambda_{j}}^{2}\right)^{w_{j}}}}, \frac{\sqrt{2 \prod_{j=1}^{n}\left(y_{\lambda_{j}}^{2}\right)^{w_{j}}}}{\sqrt{\prod_{j=1}^{n}\left(2-y_{\lambda_{j}}^{2}\right)^{w_{j}}+\prod_{j=1}^{n}\left(y_{\lambda_{j}}^{2}\right)^{w_{j}}}}\right]}
\end{array}\right),
\end{aligned}
$$

where $w=\left(w_{1}, w_{2}, w_{3}, \ldots, w_{n}\right)^{T}$ is the weighted vector of $\lambda_{j}(j=1,2,3, \ldots, n)$, such that $w_{j} \in[0,1]$ and $\sum_{j=1}^{n} w_{j}=$ 1 .

Definition 6 [32] Let $\lambda_{j}(j=1,2,3, \ldots, n)$ be a collection of IVPFVs, then IVPFEOWA operator can be defined as:

$\operatorname{IVPFEOWA}_{w}\left(\lambda_{1}, \lambda_{2}, \lambda_{3}, \ldots, \lambda_{n}\right)$

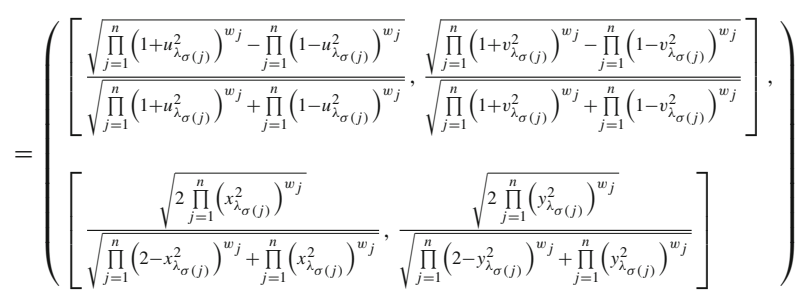

where $(\sigma(1), \sigma(2), \sigma(3), \ldots, \sigma(n))$ is a permutation of $(1,2$, $3, \ldots, n)$ such that $\sigma(j) \leq \sigma(j-1)$ for all $j$ and $w=$ $\left(w_{1}, w_{2}, w_{3}, \ldots, w_{n}\right)^{T}$ is the weighted vector of $\lambda_{\sigma(j)}(j=$ $1,2,3, \ldots, n)$ such that $w_{j} \in[0,1]$ and $\sum_{j=1}^{n} w_{j}=1$.

\section{Interval-valued Pythagorean fuzzy Einstein hybrid weighted averaging aggregation operator}

In this section, we introduce the notion of interval-valued Pythagorean fuzzy Einstein hybrid weighted averaging aggregation operator. We also discuss some desirable properties such as idempotency, boundedness and monotonicity.

Definition 7 An interval-valued Pythagorean fuzzy Einstein hybrid weighted averaging operator of dimension $n$ is a mapping IVPFEHWA : $\Theta^{n} \rightarrow \Theta$, which has associated vector $w=\left(w_{1}, w_{2}, w_{3}, \ldots, w_{n}\right)^{T}$, such that $w_{j} \in[0,1]$ and $\sum_{j=1}^{n} w_{j}=1$. Furthermore 
$\operatorname{IVPFEHWA}_{\omega, w}\left(\lambda_{1}, \lambda_{2}, \lambda_{3}, \ldots, \lambda_{n}\right)$

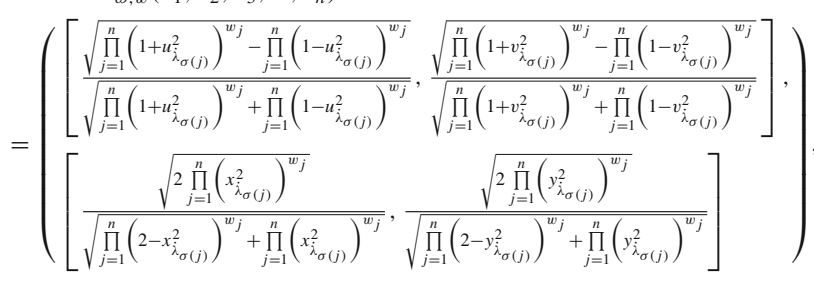

where $\dot{\lambda}_{\sigma(j)}$ is the $j$ th largest of the weighted interval-valued Pythagorean fuzzy values, $\dot{\lambda}_{\sigma(j)}\left(\dot{\lambda}_{\sigma(j)}=n \omega_{j} \lambda_{j}\right)$. $\omega=$ $\left(\omega_{1}, \omega_{2}, \omega_{3}, \ldots, \omega_{n}\right)^{T}$ is the weighted vector of $\lambda_{j}(j=1,2$, $3, \ldots, n)$ such that $\omega_{j} \in[0,1], \sum_{j=1}^{n} \omega_{j}=1$, and $n$ is the balancing coefficient, which plays a role of balance. If the vector $w=\left(w_{1}, w_{2}, w_{3}, \ldots, w_{n}\right)^{T}$ approaches to $\left(\frac{1}{n}, \frac{1}{n}, \frac{1}{n}, \ldots, \frac{1}{n}\right)^{T}$, then the vector $\left(n \omega_{1} \lambda_{1}, n \omega_{2} \lambda_{2}, \ldots\right.$, $\left.n \omega_{n} \lambda_{n}\right)^{T}$ approaches to $\left(\lambda_{1}, \lambda_{2}, \lambda_{3}, \ldots, \lambda_{n}\right)^{T}$.

Theorem 1 Let $\lambda, \lambda_{1}, \lambda_{2}$ be the three interval-valued Pythagorean fuzzy numbers and $\delta, \delta_{1}, \delta_{2} \succ 0$, then the following conditions always hold:

1. $\lambda_{1} \oplus_{\varepsilon} \lambda_{2}=\lambda_{2} \oplus_{\varepsilon} \lambda_{1}$,

2. $\lambda_{1} \otimes_{\varepsilon} \lambda_{2}=\lambda_{2} \otimes_{\varepsilon} \lambda_{1}$,

3. $\delta\left(\lambda_{1} \oplus_{\varepsilon} \lambda_{2}\right)=\delta \lambda_{1} \oplus_{\varepsilon} \delta \lambda_{2}$,

4. $\left(\lambda_{1} \otimes_{\varepsilon} \lambda_{2}\right)^{\delta}=\left(\lambda_{1}\right)^{\delta} \otimes_{\varepsilon}\left(\lambda_{2}\right)^{\delta}$,

5. $\delta_{1}(\lambda) \oplus_{\varepsilon} \delta_{2}(\lambda)=\left(\delta_{1} \oplus_{\varepsilon} \delta_{2}\right) \lambda$,

6. $(\lambda)^{\delta_{1}} \otimes_{\varepsilon}(\lambda)^{\delta_{2}}=\lambda^{\left(\delta_{1} \otimes_{\varepsilon} \delta_{2}\right)}$.

Proof The proof is trivial, so it is omitted here.

Theorem 2 Let $\lambda_{j}=\left(\left[u_{j}, v_{j}\right],\left[x_{j}, y_{j}\right]\right)(j=1,2,3, \ldots, n)$ be a collection of IVPFVs, then their aggregated value using the IVPFEHWA operator is also an IVPFV, and

$\operatorname{IVPFEHWA}{ }_{\omega, w}\left(\lambda_{1}, \lambda_{2}, \lambda_{3}, \ldots, \lambda_{n}\right)$

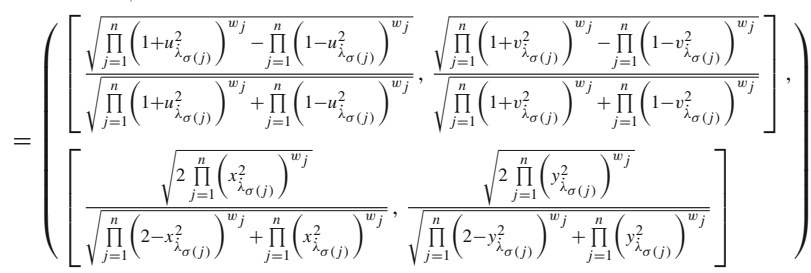

where $\dot{\lambda}_{\sigma(j)}$ is the $j$ th largest of the weighted interval-valued Pythagorean fuzzy values, $\dot{\lambda}_{\sigma(j)}\left(\dot{\lambda}_{\sigma(j)}=n \omega_{j} \lambda_{j}\right), \quad w=$ $\left(w_{1}, w_{2}, w_{2}, \ldots, w_{n}\right)^{T}$ is the weighted vector of IVPFE$H W A$, such that $w_{j} \in[0,1], \sum_{j=1}^{n} w_{j}=1$. $\omega=$ $\left(\omega_{1}, \omega_{2}, \omega_{2}, \ldots, \omega_{n}\right)^{T}$ is the weighted vector of $\lambda_{j}(j=1,2$, $3, \ldots, n)$ such that $\omega_{j} \in[0,1], \sum_{j=1}^{n} \omega_{j}=1$, and $n$ is the balancing coefficient, which plays a role of balance. If the vector $w=\left(w_{1}, w_{2}, w_{2}, \ldots, w_{n}\right)^{T}$ approaches $\left(\frac{1}{n}, \frac{1}{n}, \frac{1}{n}, \ldots, \frac{1}{n}\right)^{T}$, then the vector $\left(n w \omega \lambda_{1}\right.$, $\left.n \omega_{2} \lambda_{2}, \ldots, n \omega_{n} \lambda_{n}\right)^{T}$ approaches $\left(\lambda_{1}, \lambda_{2}, \lambda_{3}, \ldots, \lambda_{n}\right)^{T}$.

Proof We can prove this theorem by mathematical induction on $n$.

For $n=2$

$$
\begin{aligned}
& w_{1} \dot{\lambda}_{1}=\left(\left[\frac{\sqrt{\left(1+u_{\dot{\lambda}_{1}}^{2}\right)^{w_{1}}-\left(1-u_{\dot{\lambda}_{1}}^{2}\right)^{w_{1}}}}{\sqrt{\left(1+u_{\dot{\lambda}_{1}}^{2}\right)^{w_{1}}+\left(1-u_{\dot{\lambda}_{1}}^{2}\right)^{w_{1}}}},\right.\right. \\
& \left.\frac{\sqrt{\left(1+v_{\dot{\lambda}_{1}}^{2}\right)^{w_{1}}-\left(1-v_{\dot{\lambda}_{1}}^{2}\right)^{w_{1}}}}{\sqrt{\left(1+v_{\dot{\lambda}_{1}}^{2}\right)^{w_{1}}+\left(1-v_{\dot{\lambda}_{1}}^{2}\right)^{w_{1}}}}\right] \\
& {\left[\frac{\sqrt{2\left(x_{\dot{\lambda}_{1}}^{2}\right)^{w_{1}}}}{\sqrt{\left(2-x_{\dot{\lambda}_{1}}^{2}\right)^{w_{1}}+\left(x_{\dot{\lambda}_{1}}^{2}\right)^{w_{1}}}},\right.} \\
& \left.\left.\frac{\sqrt{2\left(y_{\dot{\lambda}_{1}}^{2}\right)^{w_{1}}}}{\sqrt{\left(2-y_{\dot{\lambda}_{1}}^{2}\right)^{w_{1}}+\left(y_{\dot{\lambda}_{1}}^{2}\right)^{w_{1}}}}\right]\right)
\end{aligned}
$$

and

$$
\begin{aligned}
w_{2} \dot{\lambda}_{2}= & {\left[\frac{\sqrt{\left(1+u_{\dot{\lambda}_{2}}^{2}\right)^{w_{2}}-\left(1-u_{\dot{\lambda}_{2}}^{2}\right)^{w_{2}}}}{\sqrt{\left(1+u_{\dot{\lambda}_{2}}^{2}\right)^{w_{2}}+\left(1-u_{\dot{\lambda}_{2}}^{2}\right)^{w_{2}}}},\right.} \\
& \left.\frac{\left.\sqrt{\left(1+v_{\dot{\lambda}_{2}}^{2}\right)^{w_{2}}-\left(1-v_{\dot{\lambda}_{2}}^{2}\right)^{w_{2}}}\right]}{\sqrt{\left(1+v_{\dot{\lambda}_{2}}^{2}\right)^{w_{2}}+\left(1-v_{\dot{\lambda}_{2}}^{2}\right)^{w_{2}}}}\right], \\
& {\left[\frac{\sqrt{\left(2-x_{\dot{\lambda}_{2}}^{2}\right)^{w_{2}}+\left(x_{\dot{\lambda}_{2}}^{2}\right)^{w_{2}}}}{\sqrt{\left(2-y_{2}\right.}},\right.} \\
& \left.\left.\sqrt{\sqrt{\left(2-y_{\dot{\lambda}_{2}}^{2}\right)^{w_{2}}+\left(y_{\dot{\lambda}_{2}}^{2}\right)^{w_{2}}}}\right]\right)
\end{aligned}
$$


Then

$\operatorname{IVPFEHWA}_{\omega, w}\left(\lambda_{1}, \lambda_{2}\right)$

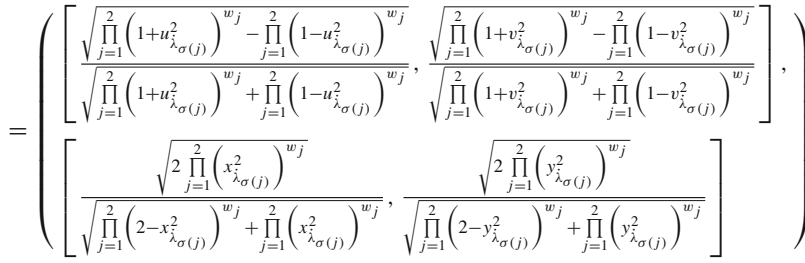

Thus, the result is true for $n=2$, now we assume that Eq. (19) holds for $n=k$. Thus

$$
\begin{aligned}
& \operatorname{IVPFEHWA}_{\omega, w}\left(\lambda_{1}, \lambda_{2}, \lambda_{3}, \ldots, \lambda_{k}\right)
\end{aligned}
$$

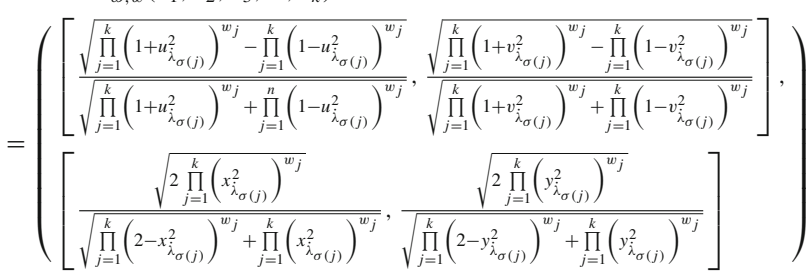

If Eq. (19) holds for $n=k$, then we show that Eq. (19) holds for $n=k+1$. Thus

$\operatorname{IVPFEHWA}_{\omega, w}\left(\lambda_{1}, \lambda_{2}, \lambda_{3}, \ldots, \lambda_{k+1}\right)$

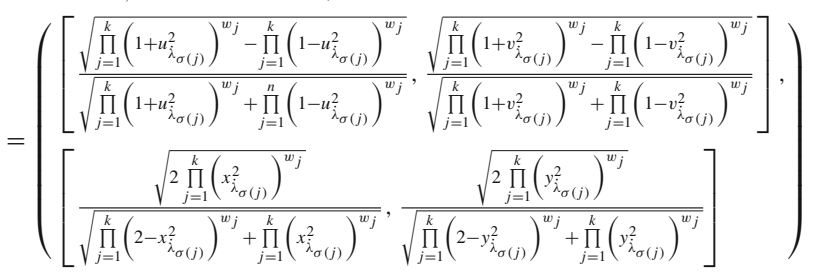$$
\oplus_{\varepsilon}\left(\begin{array}{l}
{\left[\frac{\sqrt{\left(1+u_{\dot{\lambda}_{k+1}}^{2}\right)^{w_{k+1}}-\left(1-u_{\dot{k}_{k+1}}^{2}\right)^{w_{k+1}}}}{\sqrt{\left(1+u_{\dot{\lambda}_{k+1}}^{2}\right)^{w_{k+1}}+\left(1-u_{\dot{\lambda}_{k+1}}^{2}\right)^{w_{k+1}}}}, \frac{\sqrt{\left(1+v_{\dot{\lambda}_{k+1}}^{2}\right)^{w_{k+1}}-\left(1-v_{\dot{\lambda}_{k+1}}^{2}\right)^{w_{k+1}}}}{\sqrt{\left(1+v_{\dot{\lambda}_{k+1}}^{2}\right)^{w_{k+1}}+\left(1-v_{\dot{\lambda}_{k+1}}^{2}\right)^{w k+1}}}\right],} \\
{\left[\frac{\sqrt{2\left(x_{\dot{\lambda}_{k+1}}^{2}\right)^{w_{k+1}}}}{\sqrt{\left(2-x_{\dot{\lambda}_{k+1}}^{2}\right)^{w_{k+1}}+\left(x_{\dot{\lambda}_{k+1}}^{2}\right)^{w_{k+1}}}}, \frac{\sqrt{2\left(y_{\dot{\lambda}_{k+1}^{2}}^{2}\right)^{w_{k+1}}}}{\sqrt{\left(2-y_{\dot{\lambda}_{k+1}}^{2}\right)^{w_{k+1}}+\left(y_{\dot{\lambda}_{k+1}}^{2}\right)^{w_{k+1}}}}\right]}
\end{array}\right)
$$

Let

$$
\begin{aligned}
& t_{1}=\sqrt{\prod_{j=1}^{k}\left(1+u_{\dot{\lambda}_{\sigma(j)}}^{2}\right)^{w_{j}}-\prod_{j=1}^{k}\left(1-u_{\dot{\lambda}_{\sigma(j)}}^{2}\right)^{w_{j}}} \\
& t_{2}=\sqrt{\prod_{j=1}^{k}\left(1+u_{\dot{\lambda}_{\sigma(j)}}^{2}\right)^{w_{j}}+\prod_{j=1}^{n}\left(1-u_{\dot{\lambda}_{\sigma(j)}}^{2}\right)^{w_{j}}} \\
& p_{1}=\sqrt{\prod_{j=1}^{k}\left(1+v_{\dot{\lambda}_{\sigma(j)}}^{2}\right)^{w_{j}}-\prod_{j=1}^{k}\left(1-v_{\dot{\lambda}_{\sigma(j)}}^{2}\right)^{w_{j}}} \\
& p_{2}=\sqrt{\prod_{j=1}^{k}\left(1+v_{\dot{\lambda}_{\sigma(j)}}^{2}\right)^{w_{j}}+\prod_{j=1}^{k}\left(1-v_{\dot{\lambda}_{\sigma(j)}}^{2}\right)^{w_{j}}}
\end{aligned}
$$

$$
\begin{aligned}
& m_{1}=\sqrt{\left(1+u_{\dot{\lambda}_{k+1}}^{2}\right)^{w_{k+1}}-\left(1-u_{\dot{\lambda}_{k+1}}^{2}\right)^{w_{k+1}}} \\
& m_{2}=\sqrt{\left(1+u_{\dot{\lambda}_{k+1}}^{2}\right)^{w_{k+1}}+\left(1-u_{\dot{\lambda}_{k+1}}^{2}\right)^{w_{k+1}}} \\
& a_{1}=\sqrt{\left(1+v_{\dot{\lambda}_{k+1}}^{2}\right)^{w_{k+1}}-\left(1-v_{\dot{\lambda}_{k+1}}^{2}\right)^{w_{k+1}}} \\
& a_{2}=\sqrt{\left(1+v_{\dot{\lambda}_{k+1}}^{2}\right)^{w_{k+1}}+\left(1-v_{\dot{\lambda}_{k+1}}^{2}\right)^{w k+1}}
\end{aligned}
$$$$
r_{2}=\sqrt{\prod_{j=1}^{k}\left(2-x_{\dot{\lambda}_{\sigma(j)}}^{2}\right)^{w_{j}}+\prod_{j=1}^{k}\left(x_{\dot{\lambda}_{\sigma(j)}}^{2}\right)^{w_{j}}}
$$$$
r_{1}=\sqrt{2 \prod_{j=1}^{k}\left(x_{\dot{\lambda}_{\sigma(j)}}^{2}\right)^{w_{j}}}, s_{1}=\sqrt{2 \prod_{j=1}^{k}\left(y_{\dot{\lambda}_{\sigma(j)}}^{2}\right)^{w_{j}}}
$$$$
s_{2}=\sqrt{\prod_{j=1}^{k}\left(2-y_{\dot{\lambda}_{\sigma(j)}}^{2}\right)^{w_{j}}+\prod_{j=1}^{k}\left(y_{\dot{\lambda}_{\sigma(j)}}^{2}\right)^{w_{j}}}
$$$$
b_{2}=\sqrt{\left(2-x_{\dot{\lambda}_{k+1}}^{2}\right)^{w_{k+1}}+\left(x_{\dot{\lambda}_{k+1}}^{2}\right)^{w_{k+1}}}
$$$$
b_{1}=\sqrt{2\left(x_{\dot{\lambda}_{k+1}}^{2}\right)^{w_{k+1}}}, c_{1}=\sqrt{2\left(y_{\dot{\lambda}_{k+1}}^{2}\right)^{w_{k+1}}}
$$$$
c_{2}=\sqrt{\left(2-y_{\dot{\lambda}_{k+1}}^{2}\right)^{w_{k+1}}+\left(y_{\dot{\lambda}_{k+1}}^{2}\right)^{w_{k+1}}}
$$

Now putting these values in Eq. (20), we have

$$
\begin{aligned}
& \operatorname{IVPFEHWA}_{\omega, w}\left(\lambda_{1}, \lambda_{2}, \lambda_{3}, \ldots, \lambda_{k+1}\right) \\
& =\left(\left[\frac{t_{1}}{t_{2}}, \frac{p_{1}}{p_{2}}\right],\left[\frac{r_{1}}{r_{2}}, \frac{s_{1}}{s_{2}}\right]\right) \oplus_{\varepsilon}\left(\left[\frac{m_{1}}{m_{2}}, \frac{a_{1}}{a_{2}}\right],\left[\frac{b_{1}}{b_{2}}, \frac{c_{1}}{c_{2}}\right]\right) \\
& =\left(\left[\frac{\sqrt{\left(\frac{t_{1}}{t_{2}}\right)^{2}+\left(\frac{m_{1}}{m_{2}}\right)^{2}}}{\sqrt{1+\left(\frac{t_{1}}{t_{2}}\right)^{2}\left(\frac{m_{1}}{m_{2}}\right)^{2}}}, \frac{\sqrt{\left(\frac{p_{1}}{p_{2}}\right)^{2}+\left(\frac{a_{1}}{a_{2}}\right)^{2}}}{\sqrt{1+\left(\frac{p_{1}}{p_{2}}\right)^{2}\left(\frac{a_{1}}{a_{2}}\right)^{2}}}\right]\right. \text {, } \\
& {\left[\frac{\left(\frac{r_{1}}{r_{2}}\right)\left(\frac{b_{1}}{b_{2}}\right)}{\sqrt{1+\left(1-\left(\frac{r_{1}}{r_{2}}\right)^{2}\right)\left(1-\left(\frac{b_{1}}{b_{2}}\right)^{2}\right)}},\right.} \\
& \left.\left.\frac{\left(\frac{s_{1}}{s_{2}}\right)\left(\frac{c_{1}}{c_{2}}\right)}{\sqrt{1+\left(1-\left(\frac{s_{1}}{s_{2}}\right)\right)^{2}\left(1-\left(\frac{c_{1}}{c_{2}}\right)\right)^{2}}}\right]\right) \\
& =\left(\left[\frac{\sqrt{\left(t_{1} m_{2}\right)^{2}+\left(t_{2} m_{1}\right)^{2}}}{\sqrt{\left(t_{2} m_{2}\right)^{2}+\left(t_{1} m_{1}\right)^{2}}}, \frac{\sqrt{\left(p_{1} a_{2}\right)^{2}+\left(a_{1} p_{2}\right)^{2}}}{\sqrt{\left(p_{2} a_{2}\right)^{2}+\left(p_{1} a_{1}\right)^{2}}}\right]\right. \text {, }
\end{aligned}
$$




$$
\begin{gathered}
{\left[\frac{r_{1} b_{1}}{\sqrt{2 r_{2}^{2} b_{2}^{2}+r_{1}^{2} b_{1}^{2}-r_{2}^{2} b_{1}^{2}-r_{1}^{2} b_{2}^{2}}},\right.} \\
\left.\left.\frac{s_{1} c_{1}}{\sqrt{2 s_{2}^{2} c_{2}^{2}+s_{1}^{2} c_{1}^{2}-s_{2}^{2} c_{1}^{2}-s_{1}^{2} c_{2}^{2}}}\right]\right) .
\end{gathered}
$$

Again putting the values of $\left(t_{1} m_{2}\right)^{2}+\left(t_{2} m_{1}\right)^{2},\left(t_{2} m_{2}\right)^{2}+$ $\left(t_{1} m_{1}\right)^{2},\left(p_{1} a_{2}\right)^{2}+\left(a_{1} p_{2}\right)^{2},\left(p_{2} a_{2}\right)^{2}+\left(p_{1} a_{1}\right)^{2}, r_{1} b_{1}, 2 r_{2}^{2} b_{2}^{2}+$ $r_{1}^{2} b_{1}^{2}-r_{2}^{2} b_{1}^{2}-r_{1}^{2} b_{2}^{2}, s_{1} c_{1}, 2 s_{2}^{2} c_{2}^{2}+s_{1}^{2} c_{1}^{2}-s_{2}^{2} c_{1}^{2}-s_{1}^{2} c_{2}^{2}$, in Eq. (21), then

$$
\begin{aligned}
& \operatorname{IVPFEHWA}_{\omega, w}\left(\lambda_{1}, \lambda_{2}, \lambda_{3}, \ldots, \lambda_{k+1}\right)
\end{aligned}
$$

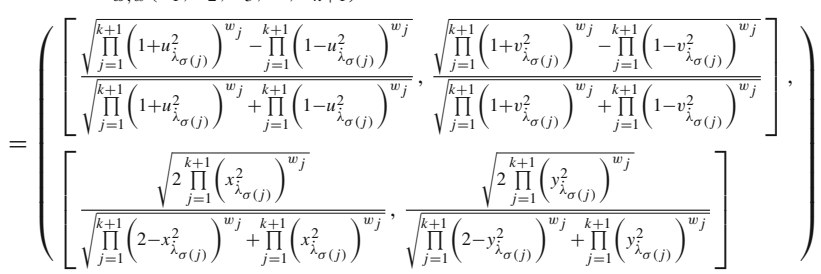

Hence, Eq. (19) holds for $n=k+1$. Thus, Eq. (19) holds for all $n$.

Remark 1 In the following, let us look $\delta \lambda$ and $\lambda^{\delta}$ some special cases of $\delta$ and $\lambda$.

1. If $\lambda=([u, v],[x, y])=([1,1]$, $[0,0])$ i. e,. $u=v=1$ and $u=v=1$, then

$$
\begin{aligned}
\lambda^{\delta}= & \left(\left[\frac{\sqrt{2\left(u^{2}\right)^{\delta}}}{\sqrt{\left(2-u^{2}\right)^{\delta}+\left(u^{2}\right)^{\delta}}}, \frac{\sqrt{2\left(v^{2}\right)^{\delta}}}{\sqrt{\left(2-v^{2}\right)^{\delta}+\left(v^{2}\right)^{\delta}}}\right],\right. \\
& {\left[\frac{\sqrt{\left(1+x^{2}\right)^{\delta}-\left(1-x^{2}\right)^{\delta}}}{\sqrt{\left(1+x^{2}\right)^{\delta}+\left(1-x^{2}\right)^{\delta}}},\right.} \\
& \left.\left.\frac{\sqrt{\left(1+y^{2}\right)^{\delta}-\left(1-y^{2}\right)^{\delta}}}{\sqrt{\left(1+y^{2}\right)^{\delta}+\left(1-y^{2}\right)^{\delta}}}\right]\right) \\
= & \left(\left[\frac{\sqrt{2(1)^{\delta}}}{\sqrt{(2-1)^{\delta}+(1)^{\delta}},} \frac{\sqrt{2(1)^{\delta}}}{\sqrt{(2-1)^{\delta}+(1)^{\delta}}}\right],\right. \\
= & {\left.\left[\frac{\sqrt{(1+0)^{\delta}-(1-0)^{\delta}}}{\sqrt{(1+0)^{\delta}+(1-0)^{\delta}}}, \frac{\sqrt{(1+0)^{\delta}-(1-0)^{\delta}}}{\sqrt{(1+0)^{\delta}+(1-0)^{\delta}}}\right]\right) } \\
& ([1,[0,0]) .
\end{aligned}
$$

Thus $\lambda^{\delta}=([1,1],[0,0])$ and $\delta \lambda=([0,0],[1,1])$.

2. If $\lambda=([u, v],[x, y])=([0,0],[1,1])$ i. e,. $u=v=0$ and $x=y=1$, then

$$
\begin{aligned}
\lambda^{\delta}= & \left(\left[\frac{\sqrt{2\left(u^{2}\right)^{\delta}}}{\sqrt{\left(2-u^{2}\right)^{\delta}+\left(u^{2}\right)^{\delta}}}, \frac{\sqrt{2\left(v^{2}\right)^{\delta}}}{\sqrt{\left(2-v^{2}\right)^{\delta}+\left(v^{2}\right)^{\delta}}}\right],\right. \\
& {\left[\frac{\sqrt{\left(1+x^{2}\right)^{\delta}-\left(1-x^{2}\right)^{\delta}}}{\sqrt{\left(1+x^{2}\right)^{\delta}+\left(1-x^{2}\right)^{\delta}}},\right.} \\
& \left.\left.\frac{\sqrt{\left(1+y^{2}\right)^{\delta}-\left(1-y^{2}\right)^{\delta}}}{\sqrt{\left(1+y^{2}\right)^{\delta}+\left(1-y^{2}\right)^{\delta}}}\right]\right) \\
= & \left(\left[\frac{\sqrt{2(0)^{\delta}}}{\left.\sqrt{(2-0)^{\delta}+(0)^{\delta}}, \frac{\sqrt{\left(2(0)^{\delta}\right.}}{\sqrt{(2-0)^{\delta}+(0)^{\delta}}}\right],}\right.\right. \\
= & {\left.\left[\frac{\sqrt{(1+1)^{\delta}-(1-1)^{\delta}}}{\sqrt{(1+1)^{\delta}+(1-1)^{\delta}}}, \frac{\sqrt{(1+1)^{\delta}-(1-1)^{\delta}}}{\sqrt{(1+1)^{\delta}+(1-1)^{\delta}}}\right]\right) } \\
& ([0,0],[1,1]) .
\end{aligned}
$$

Thus $\lambda^{\delta}=([0,0],[1,1])$ and $\delta \lambda=([1,1],[0,0])$.

3. If $\lambda=([u, v],[x, y])=([0,0],[0,0])$ i. e,. $u=v=0$ and $x=y=0$, then

$$
\begin{aligned}
\lambda^{\delta}= & \left(\left[\frac{\sqrt{2\left(u^{2}\right)^{\delta}}}{\sqrt{\left(2-u^{2}\right)^{\delta}+\left(u^{2}\right)^{\delta}}}, \frac{\sqrt{2\left(v^{2}\right)^{\delta}}}{\sqrt{\left(2-v^{2}\right)^{\delta}+\left(v^{2}\right)^{\delta}}}\right],\right. \\
& {\left[\frac{\sqrt{\left(1+x^{2}\right)^{\delta}-\left(1-x^{2}\right)^{\delta}}}{\sqrt{\left(1+x^{2}\right)^{\delta}+\left(1-x^{2}\right)^{\delta}}},\right.} \\
& \left.\left.\frac{\sqrt{\left(1+y^{2}\right)^{\delta}-\left(1-y^{2}\right)^{\delta}}}{\sqrt{\left(1+y^{2}\right)^{\delta}+\left(1-y^{2}\right)^{\delta}}}\right]\right) \\
= & \left(\left[\frac{\sqrt{2(0)^{\delta}}}{\left.\sqrt{(2-0)^{\delta}+(0)^{\delta}}, \frac{\sqrt{\left(2(0)^{\delta}\right.}}{\sqrt{(2-0)^{\delta}+(0)^{\delta}}}\right],}\right.\right. \\
= & {\left.\left[\frac{\sqrt{(1+0)^{\delta}-(1-0)^{\delta}}}{\sqrt{(1+0)^{\delta}+(1-0)^{\delta}}}, \frac{\sqrt{(1+0)^{\delta}-(1-0)^{\delta}}}{\sqrt{(1+0)^{\delta}+(1-0)^{\delta}}}\right]\right) } \\
= & ([0,0],[0,0]) .
\end{aligned}
$$

Thus $\lambda^{\delta}=([0,0],[0,0])$ and $\delta \lambda=([0,0],[0,0])$.

4. If $\delta \rightarrow 0$ and $0 \leq u, v, x, y \leq 1$, then

$$
\begin{aligned}
\lambda^{\delta}= & \left(\left[\frac{\sqrt{2\left(u^{2}\right)^{\delta}}}{\sqrt{\left(2-u^{2}\right)^{\delta}+\left(u^{2}\right)^{\delta}}}, \frac{\sqrt{2\left(v^{2}\right)^{\delta}}}{\sqrt{\left(2-v^{2}\right)^{\delta}+\left(v^{2}\right)^{\delta}}}\right],\right. \\
& {\left[\frac{\sqrt{\left(1+x^{2}\right)^{\delta}-\left(1-x^{2}\right)^{\delta}}}{\sqrt{\left(1+x^{2}\right)^{\delta}+\left(1-x^{2}\right)^{\delta}}},\right.} \\
& \left.\left.\frac{\sqrt{\left(1+y^{2}\right)^{\delta}-\left(1-y^{2}\right)^{\delta}}}{\sqrt{\left(1+y^{2}\right)^{\delta}+\left(1-y^{2}\right)^{\delta}}}\right]\right) \\
= & \left(\left[\frac{\sqrt{2\left(u^{2}\right)^{0}}}{\sqrt{\left(2-u^{2}\right)^{0}+\left(u^{2}\right)^{0}}}, \frac{\sqrt{2\left(v^{2}\right)^{0}}}{\sqrt{\left(2-v^{2}\right)^{0}+\left(v^{2}\right)^{0}}}\right],\right.
\end{aligned}
$$




$$
\begin{aligned}
& {\left[\frac{\sqrt{\left(1+x^{2}\right)^{0}-\left(1-x_{1}^{2}\right)^{0}}}{\sqrt{\left(1+x^{2}\right)^{0}+\left(1-x^{2}\right)^{0}}},\right.} \\
& \left.\left.\frac{\sqrt{\left(1+y^{2}\right)^{0}-\left(1-y^{2}\right)^{0}}}{\sqrt{\left(1+y^{2}\right)^{0}+\left(1-y^{2}\right)^{0}}}\right]\right) \\
= & ([1,1],[0,0]) .
\end{aligned}
$$

Thus $\lambda^{\delta}=([1,1],[0,0])$ and $\delta \lambda=([0,0],[1,1])$.

5. If $\delta \rightarrow+\infty$ and $0 \leq u, v, x, y \leq 1$, then

$$
\begin{aligned}
\lambda^{\delta}= & \left(\left[\frac{\sqrt{2\left(u^{2}\right)^{\delta}}}{\sqrt{\left(2-u^{2}\right)^{\delta}+\left(u^{2}\right)^{\delta}}}, \frac{\sqrt{2\left(v^{2}\right)^{\delta}}}{\sqrt{\left(2-v^{2}\right)^{\delta}+\left(v^{2}\right)^{\delta}}}\right],\right. \\
& {\left[\frac{\sqrt{\left(1+x^{2}\right)^{\delta}-\left(1-x^{2}\right)^{\delta}}}{\sqrt{\left(1+x^{2}\right)^{\delta}+\left(1-x^{2}\right)^{\delta}}},\right.} \\
& \left.\left.\frac{\sqrt{\left(1+y^{2}\right)^{\delta}-\left(1-y^{2}\right)^{\delta}}}{\sqrt{\left(1+y^{2}\right)^{\delta}+\left(1-y^{2}\right)^{\delta}}}\right]\right) \\
= & \left(\left[\frac{\sqrt{2\left(u^{2}\right)^{\infty}}}{\sqrt{\left(2-u^{2}\right)^{\infty}+\left(u^{2}\right)^{\infty}}}, \frac{\sqrt{2\left(v^{2}\right)^{\infty}}}{\sqrt{\left(2-v^{2}\right)^{\infty}+\left(u^{2}\right)^{\infty}}}\right],\right. \\
& {\left[\frac{\sqrt{\left(1+x^{2}\right)^{\infty}-\left(1-x^{2}\right)^{\infty}}}{\sqrt{\left(1+x^{2}\right)^{\infty}+\left(1-x^{2}\right)^{\infty}}},\right.} \\
& \left.\left.\frac{\sqrt{\left(1+y^{2}\right)^{\infty}-\left(1-y^{2}\right)^{\infty}}}{\sqrt{\left(1+y^{2}\right)^{\infty}+\left(1-y^{2}\right)^{\infty}}}\right]\right)=([[0,0], 1,1]) .
\end{aligned}
$$

Thus, $\lambda^{\delta}=([0,0],[1,1])$ and $\delta \lambda=([1,1],[0,0])$.

6. If $\delta=1$ and $0 \leq u, v, x, y \leq 1$, then

$$
\begin{aligned}
\lambda^{\delta}= & \left(\left[\frac{\sqrt{2\left(u^{2}\right)^{\delta}}}{\sqrt{\left(2-u^{2}\right)^{\delta}+\left(u^{2}\right)^{\delta}}}, \frac{\sqrt{2\left(v^{2}\right)^{\delta}}}{\sqrt{\left(2-v^{2}\right)^{\delta}+\left(v^{2}\right)^{\delta}}}\right],\right. \\
& {\left[\frac{\sqrt{\left(1+x^{2}\right)^{\delta}-\left(1-x^{2}\right)^{\delta}}}{\sqrt{\left(1+x^{2}\right)^{\delta}+\left(1-x^{2}\right)^{\delta}}},\right.} \\
& \left.\left.\frac{\sqrt{\left(1+y^{2}\right)^{\delta}-\left(1-y^{2}\right)^{\delta}}}{\sqrt{\left(1+y^{2}\right)^{\delta}+\left(1-y^{2}\right)^{\delta}}}\right]\right) \\
= & \left(\left[\frac{\sqrt{2\left(u^{2}\right)^{1}}}{\sqrt{\left(2-u^{2}\right)^{1}+\left(u^{2}\right)^{1}}}, \frac{\sqrt{2\left(v^{2}\right)^{1}}}{\sqrt{\left(2-v^{2}\right)^{1}+\left(u^{2}\right)^{1}}}\right],\right. \\
& {\left[\frac{\sqrt{\left(1+x^{2}\right)^{1}-\left(1-x^{2}\right)^{1}}}{\sqrt{\left(1+x^{2}\right)^{1}+\left(1-x^{2}\right)^{1}}},\right.} \\
& \left.\left.\frac{\sqrt{\left(1+y^{2}\right)^{1}-\left(1-y^{2}\right)^{1}}}{\sqrt{\left(1+y^{2}\right)^{1}+\left(1-y^{2}\right)^{1}}}\right]\right)=\lambda .
\end{aligned}
$$

Thus, $\lambda^{\delta}=\lambda$ and $\delta \lambda=\lambda$.
Lemma 1 [6] Let $\lambda_{j} \succ 0, w_{j} \succ 0(j=1,2,3, \ldots, n)$ and $\sum_{j=1}^{n} w_{j}=1$, then

$$
\prod_{j=1}^{n}\left(\lambda_{j}\right)^{w_{j}} \leq \sum_{j=1}^{n} w_{j} \lambda_{j},
$$

where the equality holds if and only if $\lambda_{1}=\lambda_{2}=\cdots=\lambda_{n}$.

Theorem 3 Let $\lambda_{j}=\left(\left[u_{j}, v_{j}\right],\left[x_{j}, y_{j}\right]\right)(j=1,2,3, \ldots, n)$ be a collection of IVPFVs, where the $w=\left(w_{1}, w_{2}, w_{3}, \ldots\right.$, $\left.w_{n}\right)^{T}$ is the weighted vector of IVPFEHWA and IVPFHWA, such that $w_{j} \in[0,1]$ and $\sum_{j=1}^{n} w_{j}=1 . \omega=\left(\omega_{1}, \omega_{2}, \omega_{2}, \ldots\right.$, $\left.\omega_{n}\right)^{T}$ is the weighted vector of $\lambda_{j}(j=1,2,3, \ldots, n)$ such that $\omega_{j} \in[0,1], \sum_{j=1}^{n} \omega_{j}=1$, then

$$
\begin{aligned}
& \quad \operatorname{IVPFEHWA}_{\omega, w}\left(\lambda_{1}, \lambda_{2}, \lambda_{3}, \ldots, \lambda_{n}\right) \\
& \quad \leq \operatorname{IVPFHWA} \omega, w \\
& \quad\left(\lambda_{1}, \lambda_{2}, \lambda_{3}, \ldots, \lambda_{n}\right) .
\end{aligned}
$$

Proof Straight forward.

Theorem 4 Idempotency: If $\dot{\lambda}_{\sigma(j)}=\dot{\lambda}$ for all $j(j=1,2$, $3, \ldots, n)$, where $\lambda=([u, v],[x, y])$, then

$\operatorname{IVPFEHWA} A_{\omega, w}\left(\lambda_{1}, \lambda_{2}, \lambda_{3}, \ldots, \lambda_{n}\right)=\dot{\lambda}$.

Proof Since $\dot{\lambda}_{\sigma(j)}=\dot{\lambda}$ for all $j$, then we have

$\operatorname{IVPFEHWA}_{\omega, w}\left(\lambda_{1}, \lambda_{2}, \lambda_{3}, \ldots, \lambda_{n}\right)$

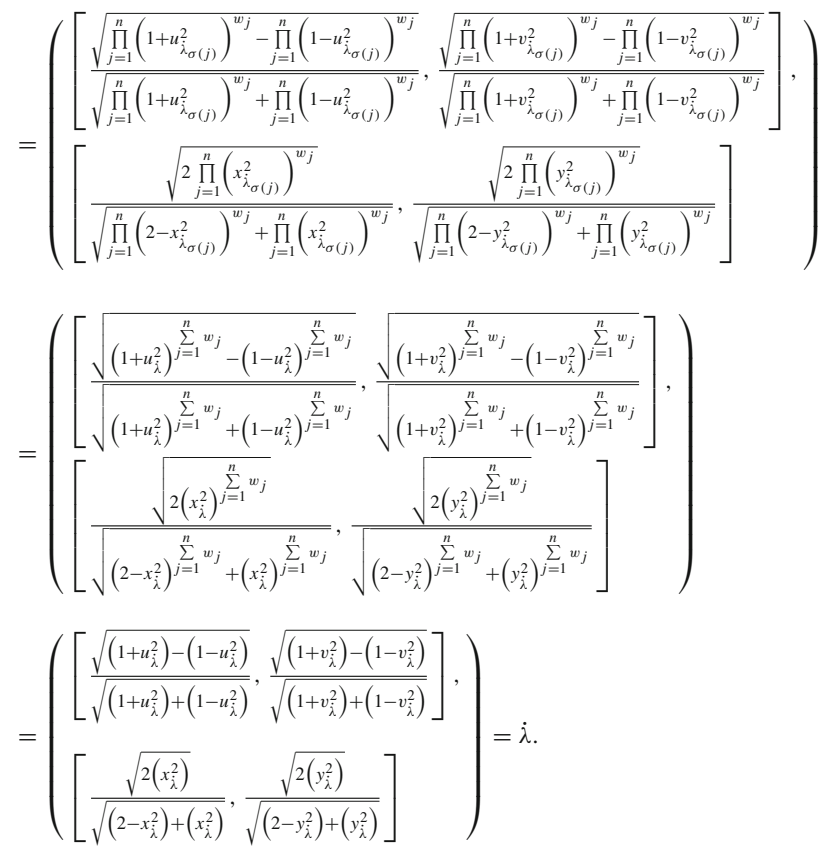

The proof is completed. 
Theorem 5 Boundedness: Let $\lambda_{j}=\left(\left[u_{\lambda_{j}}, v_{\lambda_{j}}\right],\left[x_{\lambda_{j}}, y_{\lambda_{j}}\right]\right)$ $(j=1,2,3, \ldots, n)$ be a collection of IVPFNs, then

$\dot{\lambda}_{\min } \leq \operatorname{IVPFEHWA} A_{\omega, w}\left(\lambda_{1}, \lambda_{2}, \lambda_{3}, \ldots, \lambda_{n}\right) \leq \dot{\lambda}_{\max }$,

$\dot{\lambda}_{\max }=\max _{j}\left(\dot{\lambda}_{\sigma(j)}\right)$,

$\dot{\lambda}_{\min }=\min _{j}\left(\dot{\lambda}_{\sigma(j)}\right)$.

Proof Proof is easy so it is omitted here.

Theorem 6 Monotonicity: If $\lambda_{j} \leq \lambda_{j}^{*}$ for all $j(j=$ $1,2,3, \ldots$,

$n)$, then

$$
\begin{aligned}
& \quad \operatorname{IVPFEHWA}{ }_{\omega, w}\left(\lambda_{1}, \lambda_{2}, \lambda_{3}, \ldots, \lambda_{n}\right) \\
& \quad \leq \operatorname{IVPFEHWA}{ }_{\omega, w}\left(\lambda_{1}^{*}, \lambda_{2}^{*}, \lambda_{3}^{*}, \ldots, \lambda_{n}^{*}\right) .
\end{aligned}
$$

Proof As we know that.

$$
\begin{aligned}
& \operatorname{IVPFEHWA}_{\omega, w}\left(\lambda_{1}, \lambda_{2}, \lambda_{3}, \ldots, \lambda_{n}\right) \\
& \quad=w_{1} \dot{\lambda}_{\sigma(1)} \oplus_{\varepsilon} w_{2} \dot{\lambda}_{\sigma(2)} \oplus_{\varepsilon} w_{3} \dot{\lambda}_{\sigma(3)} \oplus_{\varepsilon} \cdots \oplus_{\varepsilon} w_{n} \dot{\lambda}_{\sigma(n)},
\end{aligned}
$$

and

$$
\begin{aligned}
& \operatorname{IVPFEHWA}_{\omega, w}\left(\lambda_{1}^{*}, \lambda_{2}^{*}, \lambda_{3}^{*}, \ldots, \lambda_{n}^{*}\right) \\
& \quad=w_{1} \dot{\lambda}_{\sigma(1)}^{*} \oplus_{\varepsilon} w_{2} \dot{\lambda}_{\sigma(2)}^{*} \oplus_{\varepsilon} w_{3} \dot{\lambda}_{\sigma(3)}^{*} \oplus_{\varepsilon} \cdots \oplus_{\varepsilon} w_{n} \dot{\lambda}_{\sigma(n)}^{*} .
\end{aligned}
$$

Since $\lambda_{j} \leq \lambda_{j}^{*}$ for all $j$, thus Eq. (28) always holds.

Theorem 7 Interval-valued Pythagorean fuzzy Einstein weighted averaging operator is a special case of the intervalvalued Pythagorean fuzzy Einstein hybrid weighted averaging operator.

Proof Let $\omega=\left(\frac{1}{n}, \frac{1}{n}, \frac{1}{n}, \ldots, \frac{1}{n},\right)^{T}$, then we have

$$
\begin{aligned}
& \operatorname{IVPFEHWA}_{\omega, w}\left(\lambda_{1}, \lambda_{2}, \lambda_{3}, \ldots, \lambda_{n}\right) \\
& \quad=w_{1} \dot{\lambda}_{\sigma(1)} \oplus_{\varepsilon} w_{2} \dot{\lambda}_{\sigma(2)} \oplus_{\varepsilon} \cdots \oplus_{\varepsilon} w_{n} \dot{\lambda}_{\sigma(n)} \\
& =\frac{1}{n}\left(\dot{\lambda}_{\sigma(1)} \oplus_{\varepsilon} \dot{\lambda}_{\sigma(2)} \oplus_{\varepsilon} \cdots \oplus_{\varepsilon} \dot{\lambda}_{\sigma(n)}\right) \\
& =\frac{1}{n}\left(n \omega_{1} \lambda_{1} \oplus_{\varepsilon} n \omega_{2} \lambda_{2} \oplus_{\varepsilon} \cdots \oplus_{\varepsilon} n \omega_{n} \lambda_{n}\right) \\
& =\omega_{1} \lambda_{1} \oplus_{\varepsilon} \omega_{2} \lambda_{2} \oplus_{\varepsilon} \cdots \oplus_{\varepsilon} \omega_{n} \lambda_{n} \\
& =\operatorname{IVPFEWA}_{w}\left(\lambda_{1}, \lambda_{2}, \lambda_{3}, \ldots, \lambda_{n}\right) .
\end{aligned}
$$

The proof is completed.

Theorem 8 Interval-valued Pythagorean fuzzy Einstein ordered weighted averaging operator is a special case of the interval-valued Pythagorean fuzzy Einstein hybrid weighted averaging operator.
Proof Let $w=\left(\frac{1}{n}, \frac{1}{n}, \frac{1}{n}, \ldots, \frac{1}{n},\right)^{T}$, and $\dot{\lambda}_{\sigma(j)}=\lambda_{\sigma(j)}$, then we have

$$
\begin{aligned}
& \operatorname{IVPFEHWA}_{\omega, w}\left(\lambda_{1}, \lambda_{2}, \lambda_{3}, \ldots, \lambda_{n}\right) \\
& \quad=w_{1} \dot{\lambda}_{\sigma(1)} \oplus_{\varepsilon} w_{2} \dot{\lambda}_{\sigma(2)} \oplus_{\varepsilon} \cdots \oplus_{\varepsilon} w_{n} \dot{\lambda}_{\sigma(n)} \\
& \quad=w_{1} \lambda_{\sigma(1)} \oplus_{\varepsilon} w_{2} \lambda_{\sigma(2)} \oplus_{\varepsilon} \cdots \oplus_{\varepsilon} w_{n} \lambda_{\sigma(n)} \\
& \quad=\operatorname{IVPFEOWA}_{w}\left(\lambda_{1}, \lambda_{2}, \lambda_{3}, \ldots, \lambda_{n}\right) .
\end{aligned}
$$

The proof completed.

\section{An approach to multiple attribute group decision-making problems based on interval-valued Pythagorean fuzzy information}

Algorithm Let $X=\left\{X_{1}, X_{2}, X_{3}, \ldots, X_{m}\right\}$ be a finite set of $m$ alternatives and $C=\left\{C_{1}, C_{2}, C_{3}, \ldots, C_{n}\right\}$ be a finite set of $n$ attributes. Suppose the grade of the alternatives $X_{i}(i=$ $1,2,3, \ldots, m)$ on attribute $C_{j}(j=1,2,3, \ldots, n)$ given by decision makers is interval-valued Pythagorean fuzzy numbers. Let $D=\left\{D_{1}, D_{2}, D_{3}, \ldots, D_{k}\right\}$ be the set of $k$ decision makers, and let $w=\left(w_{1}, w_{2}, w_{3}, \ldots, w_{n}\right)^{T}$ be the weighted vector of the attributes $C_{j}(j=1,2,3, \ldots, n)$, such that $w_{j} \in[0,1], \sum_{j=1}^{n} w_{j}=1$, and let $\omega=$ $\left(\omega_{1}, \omega_{2}, \omega_{3}, \ldots, \omega_{k}\right)^{T}$ be the weighted vector of the decision makers $D^{s}(s=1,2,3, \ldots, k)$, such that $\omega_{s} \in[0,1]$ and $\sum_{s=1}^{k} \omega_{s}=1$. Let $D=\left(a_{j i}\right)=\left\langle\left[u_{j i}, v_{j i}\right],\left[x_{j i}, y_{j i}\right]\right\rangle(i=$ $1,2,3, \ldots, m, j=1,2,3, \ldots, n)$ where $\left[u_{j i}, v_{j i}\right]$ indicates the interval degree that the alternative $X_{i}(i=1,2,3, \ldots, m)$ satisfies the attribute $C_{j}(j=1,2,3, \ldots, n)$ and $\left[x_{j i}, y_{j i}\right]$ indicates the interval degree that the alternative $X_{i}(i=$ $1,2,3, \ldots, m)$ does not satisfy the attribute $C_{j}(j=1,2,3$, $\ldots, n)$, And also $\left[u_{j i}, v_{j i}\right] \in[0,1],\left[x_{j i}, y_{j i}\right] \in[0,1]$ with condition $0 \leq\left(v_{j i}\right)^{2}+\left(y_{j i}\right)^{2} \leq 1,(i=1,2,3, \ldots, m, j=$ $1,2,3, \ldots, n)$. This method has the following steps.

Step 1 Utilize the given information in the form of matrices, $D^{s}=\left[a_{j i}^{(s)}\right]_{n \times m}(s=1,2,3, \ldots, k)$.

Step 2 If the criteria have two types, such as benefit criteria and cost criteria, then the interval-valued Pythagorean fuzzy decision matrices, $D^{s}=\left[a_{j i}^{(s)}\right]_{n \times m}$ $(s=1,2,3, \ldots, k)$ can be converted into the normalized interval-valued Pythagorean fuzzy decision matrices, $R^{s}=\left[r_{j i}^{(s)}\right]_{n \times m}(s=1,2,3, \ldots, n)$, where $r_{j i}^{(s)}=\left\{\begin{array}{l}a_{j i}^{(s)}, \text { for benefit criteria } C_{j} \\ \bar{a}_{j i}^{(s)}, \text { for cost criteria } C_{j},\end{array}\left(\begin{array}{l}j=1,2,3, \ldots, n \\ i=1,2,3, \ldots, m\end{array}\right)\right.$, 
and $\bar{a}_{j i}^{(s)}$ is the complement of $\alpha_{j i}^{s}$. If all the criteria have the same type, then there is no need of normalization.

Step 3 Utilize the IVPFEWA operator to aggregate all the individual normalized interval-valued Pythagorean fuzzy decision matrices, $R^{s}=\left[r_{j i}^{(s)}\right]_{n \times m}(s=1,2$, $3, \ldots, k)$ into a single interval-valued Pythagorean fuzzy decision-matrix, $R=\left[r_{j i}\right]_{n \times m}$, where $r_{j i}=$ $\left\langle\left[u_{j i}, v_{j i}\right],\left[x_{j i}, y_{j i}\right]\right\rangle$.

Step 4 In this step, we calculate $\dot{r}_{j i}=n w_{j} r_{j i}$.

Step 5 Calculate the scores function of $\dot{r}_{j i}(i=1,2,3, \ldots$, $m, j=1,2,3, \ldots, n)$. If there is no difference between two or more than two scores, then we must find out the accuracy degrees of the collective overall preference values.

Step 6 Utilize the IVPFEHWA operator to aggregate all preference values.

Step 7 Arrange the scores of the all alternatives in the form of descending order and select that alternative which has the highest score function.

\section{Illustrative example}

Suppose in Hazara University, the IT department wants to select a new information system for the purpose of the best productivity. After the first selection, there are only three $X_{i}(i=1,2,3)$ alternatives have been short listed. There are three experts $D^{s}(s=1,2,3)$ from a group to act as decision makers, whose weight vector is $\omega=(0.2,0.3,0.5)^{T}$. There are many factors that must be considered while selecting the most suitable system, but here, we have consider only the following four criteria, whose weighted vector is $w=$ $(0.1,0.2,0.3,0.4)^{T}$

1. $C_{1}$ : Costs of hardware.

2. $C_{2}$ : Support of the organization.

3. $C_{3}$ : Effort to transform from current systems.

4. $C_{4}$ : Outsourcing software developer reliability,

where $C_{1}, C_{3}$, are cost type criteria and $C_{2}, C_{4}$ are benefit type criteria, i.e., the attributes have two types of criteria; thus, we must change the cost type criteria into benefit type criteria.

Step 1 Construct the decision-making matrices (Tables 1, 2 and 3 ).

Step 2 Construct the normalized decision making matrices (Tables 4, 5 and 6).

Step 3 Utilize the IVPFEWA operator to aggregate all the individual normalized interval-valued Pythagorean fuzzy decision matrices, $R^{s}=\left[r_{j i}^{(s)}\right]_{n \times m}$ into a single interval-valued Pythagorean fuzzy decision matrix, $R=\left[r_{j i}\right]_{n \times m}$ (Table 7).
Table 1 Interval-valued Pythagorean fuzzy decision matrix of $\mathrm{D}^{1}$

Table 2 Interval-valued Pythagorean fuzzy decision matrix of $\mathrm{D}^{2}$

Table 3 Interval-valued Pythagorean fuzzy decision matrix of $\mathrm{D}^{3}$

\begin{tabular}{llll}
\hline & $X_{1}$ & $X_{2}$ & $X_{3}$ \\
\hline$C_{1}$ & $([0.5,0.8],[0.3,0.4])$ & $([0.6,0.7],[0.3,0.6])$ & $([0.3,0.7],[0.3,0.5])$ \\
$C_{2}$ & $([0.3,0.5],[0.6,0.7])$ & $([0.3,0.7],[0.2,0.6])$ & $([0.3,0.6],[0.4,0.7])$ \\
$C_{3}$ & $([0.5,0.7],[0.3,0.7])$ & $([0.5,0.6],[0.3,0.7])$ & $([0.2,0.6],[0.3,0.7])$ \\
$C_{4}$ & $([0.3,0.6],[0.6,0.7])$ & $([0.6,0.5],[0.2,0.7])$ & $([0.3,0.4],[0.5,0.6])$ \\
\hline
\end{tabular}

\begin{tabular}{llll}
\hline & $X_{1}$ & $X_{2}$ & $X_{3}$ \\
\hline$C_{1}$ & $([0.5,0.6],[0.3,0.5])$ & $([0.5,0.7],[0.3,0.6])$ & $([0.2,0.8],[0.3,0.4])$ \\
$C_{2}$ & $([0.3,0.4],[0.6,0.8])$ & $([0.3,0.8],[0.2,0.6])$ & $([0.3,0.6],[0.3,0.7])$ \\
$C_{3}$ & $([0.4,0.5],[0.3,0.8])$ & $([0.5,0.7],[0.3,0.6])$ & $([0.2,0.6],[0.3,0.8])$ \\
$C_{4}$ & $([0.3,0.6],[0.5,0.7])$ & $([0.3,0.4],[0.2,0.8])$ & $([0.3,0.5],[0.5,0.7])$ \\
\hline
\end{tabular}

\begin{tabular}{llll}
\hline & $X_{1}$ & $X_{2}$ & $X_{3}$ \\
\hline$C_{1}$ & $([0.3,0.8],[0.5,0.6])$ & $([0.3,0.5],[0.5,0.7])$ & $([0.2,0.4],[0.5,0.7])$ \\
$C_{2}$ & $([0.5,0.7],[0.3,0.4])$ & $([0.4,0.6],[0.5,0.8])$ & $([0.5,0.7],[0.2,0.5])$ \\
$C_{3}$ & $([0.3,0.6],[0.4,0.6])$ & $([0.3,0.5],[0.5,0.6])$ & $([0.2,0.8],[0.4,0.6])$ \\
$C_{4}$ & $([0.5,0.7],[0.3,0.4])$ & $([0.5,0.7],[0.2,0.4])$ & $([0.5,0.6],[0.3,0.5])$ \\
\hline
\end{tabular}


Table 4 Normalized Pythagorean fuzzy decision matrix $\mathrm{R}^{1}$

Table 5 Normalized Pythagorean fuzzy decision matrix $\mathrm{R}^{2}$

Table 6 Normalized Pythagorean fuzzy decision matrix $\mathrm{R}^{3}$

\begin{tabular}{llll}
\hline & $X_{1}$ & $X_{2}$ & $X_{3}$ \\
\hline$C_{1}$ & $([0.3,0.4],[0.5,0.8])$ & $([0.3,0.6],[0.6,0.7])$ & $([0.3,0.5],[0.3,0.7])$ \\
$C_{2}$ & $([0.3,0.5],[0.6,0.7])$ & $([0.3,0.7],[0.2,0.6])$ & $([0.3,0.6],[0.4,0.7])$ \\
$C_{3}$ & $([0.3,0.7],[0.5,0.7])$ & $([0.3,0.7],[0.5,0.6])$ & $([0.3,0.7],[0.2,0.6])$ \\
$C_{4}$ & $([0.3,0.6],[0.6,0.7])$ & $([0.6,0.5],[0.2,0.7])$ & $([0.3,0.4],[0.5,0.6])$ \\
\hline
\end{tabular}

\begin{tabular}{llll}
\hline & $X_{1}$ & $X_{2}$ & $X_{3}$ \\
\hline$C_{1}$ & $([0.3,0.5],[0.5,0.6])$ & $([0.3,0.6],[0.5,0.7])$ & $([0.3,0.4],[0.2,0.8])$ \\
$C_{2}$ & $([0.3,0.4],[0.6,0.8])$ & $([0.3,0.8],[0.2,0.6])$ & $([0.3,0.6],[0.3,0.7])$ \\
$C_{3}$ & $([0.3,0.8],[0.4,0.5])$ & $([0.3,0.6],[0.5,0.7])$ & $([0.3,0.8],[0.2,0.6])$ \\
$C_{4}$ & $([0.3,0.6],[0.5,0.7])$ & $([0.3,0.4],[0.2,0.8])$ & $([0.3,0.5],[0.5,0.7])$ \\
\hline
\end{tabular}

\begin{tabular}{llll}
\hline & $X_{1}$ & $X_{2}$ & $X_{3}$ \\
\hline$C_{1}$ & $([0.5,0.6],[0.3,0.8])$ & $([0.5,0.7],[0.3,0.5])$ & $([0.5,0.7],[0.2,0.4])$ \\
$C_{2}$ & $([0.5,0.7],[0.3,0.4])$ & $([0.4,0.6],[0.5,0.8])$ & $([0.5,0.7],[0.2,0.5])$ \\
$C_{3}$ & $([0.4,0.6],[0.3,0.6])$ & $([0.5,0.6],[0.3,0.5])$ & $([0.4,0.6],[0.2,0.8])$ \\
$C_{4}$ & $([0.5,0.7],[0.3,0.4])$ & $([0.5,0.7],[0.2,0.4])$ & $([0.5,0.6],[0.3,0.5])$ \\
\hline
\end{tabular}

Table 7 Collective interval-valued Pythagorean fuzzy decision matrix R

\begin{tabular}{llll}
\hline & $X_{1}$ & $X_{2}$ & $X_{3}$ \\
\hline$C_{1}$ & $([0.413,0.537],[0.389,0.738])$ & $([0.413,0.653],[0.405,0.595])$ & $([0.413,0.593],[0.216,0.562])$ \\
$C_{2}$ & $([0.413,0.593],[0.429,0.563])$ & $([0.352,0.692],[0.320,0.697])$ & $([0.413,0.653],[0.260,0.595])$ \\
$C_{3}$ & $([0.352,0.692],[0.363,0.587])$ & $([0.413,0.622],[0.389,0.576])$ & $([0.352,0.692],[0.200,0.697])$ \\
$C_{4}$ & $([0.413,0.653],[0.405,0.536])$ & $([0.475,0.593],[0.200,0.563])$ & $([0.413,0.537],[0.389,0.576])$ \\
\hline
\end{tabular}

Step 4 Calculate $\dot{\lambda}_{j i}=n w \lambda_{j i}$.

$$
\begin{aligned}
& \dot{\lambda}_{11}=([0.262,0.343],[0.733,0.897]), \\
& \dot{\lambda}_{21}=([0.370,0.534],[0.523,0.645]) \\
& \dot{\lambda}_{31}=([0.385,0.745],[0.281,0.513]), \\
& \dot{\lambda}_{41}=([0.518,0.788],[0.201,0.329]) \\
& \dot{\lambda}_{12}=([0.262,0.424],[0.742,0.837]), \\
& \dot{\lambda}_{22}=([0.315,0.628],[0.420,0.757]) \\
& \dot{\lambda}_{32}=([0.452,0.665],[0.307,0.501]), \\
& \dot{\lambda}_{42}=([0.593,0.726],[0.061,0.359]) \\
& \dot{\lambda}_{13}=([0.262,0.382],[0.605,0.823]), \\
& \dot{\lambda}_{23}=([0.370,0.590],[0.357,0.672]) \\
& \dot{\lambda}_{33}=([0.385,0.745],[0.136,0.638]), \\
& \dot{\lambda}_{43}=([0.518,0.664],[0.188,0.374]) .
\end{aligned}
$$

Step 5 Calculate the score functions (Table 8).

$$
\begin{aligned}
s\left(\dot{\lambda}_{11}\right) & =-0.57, s\left(\dot{\lambda}_{21}\right)=-0.13, s\left(\dot{\lambda}_{31}\right) \\
& =0.18, s\left(\dot{\lambda}_{41}\right)=0.37 \\
s\left(\dot{\lambda}_{12}\right) & =-0.50, s\left(\dot{\lambda}_{22}\right)=-0.12, s\left(\dot{\lambda}_{32}\right) \\
& =0.15, s\left(\dot{\lambda}_{42}\right)=0.37 \\
s\left(\dot{\lambda}_{13}\right) & =-0.41, s\left(\dot{\lambda}_{23}\right)=-0.04, s\left(\dot{\lambda}_{33}\right) \\
& =0.13, s\left(\dot{\lambda}_{43}\right)=0.26 .
\end{aligned}
$$

Step 6 Utilize the IVPFEHWA aggregation operator to aggregate all preference values.

$$
r_{1}=([0.354,0.567],[0.550,0.674])
$$


Table 8 Pythagorean fuzzy hybrid decision matrix $\mathrm{R}$

\begin{tabular}{llll}
\hline & $X_{1}$ & $X_{2}$ & $X_{3}$ \\
\hline$C_{1}$ & $([0.518,0.788],[0.201,0.329])$ & $([0.593,0.726],[0.061,0.359])$ & $([0.518,0.664],[0.188,0.374])$, \\
$C_{2}$ & $([0.385,0.745],[0.281,0.513])$ & $([0.525,0.665],[0.307,0.501])$ & $([0.585,0.745],[0.136,0.638])$, \\
$C_{3}$ & $([0.370,0.534],[0.523,0.645])$ & $([0.315,0.628],[0.420,0.757])$ & $([0.370,0.590],[0.357,0.672])$, \\
$C_{4}$ & $([0.262,0.343],[0.733,0.897])$ & $([0.262,0.424],[0.742,0.837])$ & $([0.262,0.382],[0.605,0.823])$. \\
\hline
\end{tabular}

$$
\begin{aligned}
& r_{2}=([0.367,0.581],[0.422,0.686]) \\
& r_{3}=([0.354,0.571],[0.347,0.695]) .
\end{aligned}
$$

Step 7 Calculate the score functions.

$$
s\left(r_{1}\right)=-0.154, s\left(r_{1}\right)=-0.088, s\left(r_{1}\right)=-0.076 .
$$

Step 8 Arrange the scores of the all alternatives in the form of descending order and select that alternative which has the highest score function.

$$
s\left(r_{3}\right) \succ s\left(r_{2}\right) \succ s\left(r_{1}\right)
$$

Thus, the best alternative is $X_{3}$.

\section{Conclusion}

In this paper, we have developed the notion of intervalvalued Pythagorean fuzzy Einstein hybrid weighted averaging aggregation operator along with their some desirable properties such as idempotency, boundedness, and monotonicity. Actually interval-valued Pythagorean fuzzy Einstein weighted averaging aggregation operator weights only the Pythagorean fuzzy arguments and interval-valued Pythagorean fuzzy Einstein ordered weighted averaging aggregation operator weights only the ordered positions of the Pythagorean fuzzy arguments instead of weighting the Pythagorean fuzzy arguments themselves. To overcome these limitations, we have introduced an intervalvalued Pythagorean fuzzy Einstein hybrid weighted averaging aggregation operator, which weights both the given Pythagorean fuzzy value and its ordered position. Finally, the proposed operator has been applied to decision-making problems to show the validity, practicality and effectiveness of the new approach.

Open Access This article is distributed under the terms of the Creative Commons Attribution 4.0 International License (http://creativecomm ons.org/licenses/by/4.0/), which permits unrestricted use, distribution, and reproduction in any medium, provided you give appropriate credit to the original author(s) and the source, provide a link to the Creative Commons license, and indicate if changes were made.

\section{References}

1. Atanassov K (1986) Intuitionistic fuzzy sets. Fuzzy Sets Syst 20(1):87-96

2. Zadeh LA (1965) Fuzzy sets. Inf Control 8(3):338-353

3. Xu ZS, Yager RR (2006) Some geometric aggregation operators based on intuitionistic fuzzy sets. Int J Gen Syst 35(4):417-433

4. Xu ZS (2007) Intuitionistic fuzzy aggregation operators. IEEE Trans Fuzzy Syst 15(6):1179-1187

5. Wang W, Liu X (2011) Intuitionistic fuzzy geometric aggregation operators based on Einstein operations. Int J Intell Syst 26(11):1049-1075

6. Wang WZ, Liu XW (2012) Intuitionistic fuzzy information aggregation using Einstein operations. IEEE Trans Fuzzy Syst 20(5):923-938

7. Xu ZS (2007b) Methods for aggregating interval-valued intuitionistic fuzzy information and their application to decision-making. Control Decis 22(2):215-219 (in Chinese)

8. Xu ZS, Chen J (2007b) On geometric aggregation over intervalvalued intuitionistic fuzzy information. Fourth Int Conf Fuzzy Syst Knowl Discov FSKD 2:466-471

9. Wang W, Liu X (2013) Interval-valued intuitionistic fuzzy hybrid weighted averaging operator based on Einstein operation and its application to decision making. J Intell Fuzzy Syst 25(2):279-290

10. Wang W, Liu X (2013) The multi-attribute decision making method based on interval-valued intuitionistic fuzzy Einstein hybrid weighted geometric operator. Comput Math Appl 66(10):1845-56

11. Rahman K, Abdullah S, Jamil M, Khan MY (2018) Some generalized intuitionistic fuzzy Einstein hybrid aggregation operators and their application to multiple attribute group decision making. Int $\mathbf{J}$ Fuzzy Syst 20(5):1567-1575

12. Liao H, Xu Z (2014) Some new hybrid weighted aggregation operators under hesitant fuzzy multi-criteria decision making environment. J Intell Fuzzy Syst 26(4):1601-1617

13. Liao H, Xu Z (2014) Intuitionistic fuzzy hybrid weighted aggregation operators. Int J Intell Syst 29(11):971-993

14. Liao $\mathrm{H}, \mathrm{Xu} \mathrm{Z}$ (2015) Extended hesitant fuzzy hybrid weighted aggregation operators and their application in decision making. Soft Comput 19(9):2551-2564

15. Yu D, Liao H (2016) Visualization and quantitative research on intuitionistic fuzzy studies. J Intell Fuzzy Syst 30(6):3653-3663

16. Liu W, Liao H (2017) A bibliometric analysis of fuzzy decision research during 1970-2015. Int J Fuzzy Syst 19(1):1-14

17. Yager R.R (2013) Pythagorean fuzzy subsets. In Proc Joint IFSA World Congress and NAFIPS Annual Meeting, Edmonton, Canada, pp 57-61

18. Yager RR (2014) Pythagorean membership grades in multi-criteria decision making. IEEE Trans Fuzzy Syst 22(4):958-965

19. Yager RR, Abbasov AM (2013) Pythagorean membership grades, complex numbers and decision making. Int J Intell Syst 28(5):436452

20. Peng X, Yang Y (2015) Some results for Pythagorean fuzzy sets. Int J Intell Syst 30(11):1133-1160 
21. Garg H (2016) A new generalized Pythagorean fuzzy information aggregation using Einstein operations and its application to decision making. Int J Intell Syst 31(9):886-920

22. Garg H (2017) Generalized Pythagorean fuzzy geometric aggregtion operators using Einstein $t$-Norm and $t$-Conorm for multicriteria decision-making process. Int J Intell Syst 32:597-630. https:// doi.org/10.1002/int.21860

23. Rahman K, Abdullah S, Husain F, Ali Khan MS (2016) Approaches to Pythagorean fuzzy geometric aggregation operators. Int J Comput Sci Inf Secur IJCSIS 4(9): 174-200

24. Rahman K, Khan MSA, Ullah M, Fahmi A (2017) Multiple attribute group decision making for plant location selection with Pythagorean fuzzy weighted geometric aggregation. Operator Nucleus 54(1):66-74

25. Rahman K, Abdullah S, Husain F, Ali Khan MS, Shakeel M (2017) Pythagorean fuzzy ordered weighted geometric aggregation operator and their application to multiple attribute group decision making. J Appl Environ Biol Sci 7(4):67-83

26. Rahman K, Abdullah S, Ali Khan MS, Shakeel M (2016) Pythagorean fuzzy hybrid geometric aggregation operator and their applications to multiple attribute decision making. Int J Comput Sci Inf Secur IJCSIS 837-854

27. Rahman K, Ali A, Shakeel M, Khan MSA, Ullah Murad (2017) Pythagorean fuzzy weighted averaging aggregation operator and its application to decision making theory. Nucleus 54(3):190-196

28. Rahman K, Abdullah S, Ahmed R, Ullah Murad (2017) Pythagorean fuzzy Einstein weighted geometric aggregation operator and their application to multiple attribute group decision making. J Intell Fuzzy Syst 33(1):635-647
29. Peng X, Yang Y (2016) Fundamental properties of intervalvalued Pythagorean fuzzy aggregation operators. Int J Intell Syst 31(5):444-487

30. Rahman K, Ali Asad, Khan MSA (2018) Some interval-valued Pythagorean fuzzy weighted averaging aggregation operators and their application to multiple attribute decision making, Punjab University. J Math 50(2):113-129

31. Rahman K, Abdullah S, Shakeel M, Khan MSA, Ullah Murad (2017) Interval-valued Pythagorean fuzzy geometric aggregation operators and their application to decision making. Cogent Math 4(1):1338638. https://doi.org/10.1515/jisys-2017-0212

32. Rahman K, Abdullah S, Khan MSA (2018) Some interval-valued Pythagorean fuzzy Einstein weighted averaging aggregation operator and their application to group decision making. J Intell Syst. https://doi.org/10.1515/jisys-2017-0212

33. Rahman K, Abdullah S (2018) Generalized interval-valued Pythagorean fuzzy aggregation operators and their application to group decision making. Granul Comput. https://doi.org/10.1007/ s41066-018-0082-9

Publisher's Note Springer Nature remains neutral with regard to jurisdictional claims in published maps and institutional affiliations. 